International Journal of Computer Science \& Information Technology (IJCSIT) Vol 3, No 6, Dec 2011

\title{
Adaptation AC to Improve Wireless Access Network Dependability
}

\author{
Olfa BEN AHMED and Zied CHOUKAIR \\ Multimedia Mobile Radio Research Unit (MEDIATRON) \\ Higher School of Communication of Tunis (SUP'COM) \\ University of Carthage, Tunisia \\ olfa.benahmedesupcom.rnu.tn \\ z.cr@wanadoo.tn
}

\begin{abstract}
According to estimation of increasing mobile application's consumers. We are interested in Long Term Evaluation (LTE) network. LTE dependability depends on availability and reliability of his evolved Nodes Bases stations (eNB). One eNB is available when it is able yet to admit a new media and to support connection. And it is reliable when it is able to support handover connection. However we consider a dependable telecommunication service when the available and reliable system can provide an acceptable quality of service $(Q o S)$. There are works that given a higher QoS with lower dependability (availability and reliability) and works that given higher dependability with lowest QoS. We propose a new Admission Control scheme using preemption bandwidth from already connected application. Our preemption proceeds in a relative utility. Results of this work give improvement of dependability with acceptable QoS.
\end{abstract}

\section{KEYWORDS}

Admission Control Algorithm, LTE, Availability, Reliability, Utility Adaptation

\section{INTRODUCTION}

Telecommunication networks have been growing with exponential pace, each new day brings with it a new innovation in technologies \& services that put more demands for appropriate handling of such a large amount of information[1].In contrast to traditional switching circuit network limited to voice services, mobile telecommunication network offers more and more multimedia applications with audio, video and data services [2]. Nowadays multimedia mobiles applications are increasingly requested [3]. We present some statistics values showing the large rate of multimedia applications consumer:

- Mobile video will increase 66 times between 2009 and 2014.

- Video-on-demand (VoD) traffic will double every two and a half years through 2014.

- The sum of all forms of video (TV, video on demand, Internet, and P2P) will continue to exceed 91 percent of global consumer traffic by 2014.

- Internet video streaming and downloads are beginning to take a larger share of bandwidth, and will grow to nearly 60 percent of all consumer Internet traffic in 2014.

According to theses estimations of increasing mobile application's consumers, this work aims to ensure improvement of access system availability and reliability. The system is EPS (Evolved Paket System); his base station eNB (evolved Node Base) is available when it is able yet to 
International Journal of Computer Science \& Information Technology (IJCSIT) Vol 3, No 6, Dec 2011 admit a new media and to support connection. And it is reliable when it is able to support handover connection. However we consider a dependable telecommunication service when the available and reliable system can provide an acceptable quality of service (QoS). We start by presenting EPS and defining access system dependability. Such as Admission Control (AC) algorithm of eNB is responsible for admitting new service or supporting handover application, we analyze some existing works. Then we propose a new AC algorithm using preemption bandwidth from already connected application. Our preemption proceeds in a relative utility. Before concluding we present our experimental results.

\section{Evolved Packet System}

EPS (Evolved Packet System) architecture is composed of Core Network (CN) and Access Network(AN), where CN corresponds to the Evolved Packet Core (EPC) which comes from System Architecture Evolution (SAE). The AN, represented in figure 1, refers to E-UTRAN LTE. Long Term Evaluation (LTE) was introduced by 3GPP ( 3 rd Generation Partnership Project), in order to ensure the competitiveness of UMTS (Universal Mobile Telecommunication System) [3] [4].The EPS bearer is in charge of managing QoS. Multiple bearers can be established for a user to provide connectivity to the different media components of his. The overall network architecture with various EPS elements is detailed in [5].

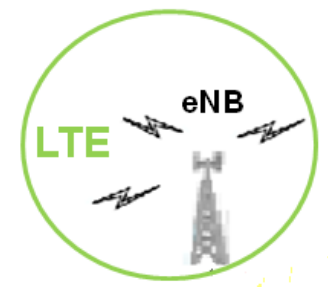

Figure 1. EPS Access Network

\section{LTE DEPENDABILITY}

To support features of multimedia applications and to provide continuous service with fewer outages and call blocking, LTE system must focus on making their networks more dependable. In competitive wireless markets, a wireless carrier must pay foremost attention to dependability to attract and retain subscribers) [6].

Dependability is the discipline that quantifies the reliance that can be placed on the service delivered by a system [7] and consists of two major aspects: availability_and reliability [8]. Additionally, there are several other aspects of dependability: Maintainability is the aptitude to undergo repairs and evolutions. Safety is dependability with respect to catastrophic failures. Integrity is an aspect of dependability that is more commonly associated with security.

LTE dependability attributes addressed in this paper include availability which will improve blocked rate and reliability which will improve outage rate in LTE system. Availability is readiness for usage, which is the probability that a system or service will be operable when needed [8]. Reliability is continuity of service that is the probability that a system or service remains operable for a specified period of time [8].

We specify our work on availability and reliability of AN which depend on availability and reliability of theirs evolved Nodes Bases stations (eNB). One eNB is available when it is able 
International Journal of Computer Science \& Information Technology (IJCSIT) Vol 3, No 6, Dec 2011 yet to admit a new media, to support connection. And it is reliable when it is able to support handover connection. To admit new media or support handover connection eNB uses Admission Control (AC) algorithm, so we propose new AC algorithm to improve dependabiliry of AN.

\section{Existing Admission CONTRol Algorithms}

Evolved Node Base station (eNB) of LTE admits a new radio bearer only if all the existing sessions and the new bearer can be guaranteed QoS according to their requirements.

- In [9] authors propose AC algorithm for LTE using the "Fractional Power Control" (FPC). To fulfill the GBR requirements the user can either be allocated larger bandwidth and transit at a lower power spectral density (PSD). The admission criterion for the new user is that the sum of the required number $(\mathrm{Ni})$ of physical resource blocks (PRBs) per transmission time interval (TTI) for the new user requesting admission as well as for existing users is less than or equal to the total number (Ntotal) of PRBs in the eNB bandwidth. This is expressed as (2)

$$
\begin{gathered}
\sum N_{i}+N_{\text {new }}<N_{\text {total }} ;(2) \\
N_{i}=\frac{G B R_{i}}{S_{i} * B W_{P R B}} \\
S_{i}=\log \left(1+S N R_{i}\right)
\end{gathered}
$$

The drawback of the FPC based AC is the increase of non admitted users in busy traffic when nodes offer the lowest PSD and then does not satisfy requirements of news users[9] . this work gives a higher QoS with lower dependability. In busy traffic the eNBs will don't be available and reliable.

- The Hybrid Admission Control (HAC) Algorithm [10] reserves a number of channels in a cell for handover calls. It accepts new calls only if there is an available bandwidth remaining after accepting the new call, is below a given threshold. HAC classifies flow on sub classes and gives a threshold for each sub class. HAC admits new requested flow for sub class if the sub class threshold is not yet full. With this algorithm, even if eNB have available resources for specific class traffic, it will not accept requests of sub classes traffics in case they do not have resources availability for their own classes.

- When total bandwidth (C) is fully used, [11] propose to preempt bandwidth that was already assigned to connected medias. This work classifies services into three classes and preempts resources from lower service priority class. The drawback of this approach is the QoS degradation of lowest priority service.

- In our paper[12], we propose a user centric AC Algorithm for UTRAN LTE. It uses two adaptations approaches. For connected users which select QR (QoS Release) the eNB minimize their number of allocated PRB proportional to the consumption of $1 / \alpha$ of their GBR requested (PGR adaptation), or their distributes the available PRB (DRA adaptation). Our solution improves the accepted number of users. But the distributed 
International Journal of Computer Science \& Information Technology (IJCSIT) Vol 3, No 6, Dec 2011 adaptation can decrease the user satisfaction. To revise this limit we use an hybrid adaptation algorithm. HA gives the maximum number between number of PRB calculated by PGR and DRA to the streaming and conversational services. For interactive and background services HA uses only DRA adaptation.

\section{IMPROVEMENT OF ACCESS DEPENDABILITY}

A LTE system's dependability expresses the expectation (trust) of its users regarding how the system meets their functional and non-functional requirements such as availability and reliability of services.

We have already mentioned that we specify our work on availability and reliability of LTE AN (access network) which depend on availability and reliability of theirs evolved Nodes Base stations (eNB). One eNB is available when it is still able to accept a new media and hence supports its connection. Moreover, it is stated as reliable when it is able to support handover connection. So we must study Admission Control (AC) algorithms to deal with dependability.

Hence we consider that a telecommunication service is dependable when the available and reliable system can provide an acceptable quality of service (QoS) according to service SLA. Some work consider a higher QoS with lower dependability (availability and reliability) whereas other workchoose to increase dependability while decreasing QoS. According to our results developed in results section, we opted for a proposal which improves dependability while providing acceptable QoS.

\subsection{Overview}

Before we develop our approach, we must present firstly some terms related to our proposal:

- A "bearer" is the basic enabler for traffic separation that is in our approach, it gives treatment differentiation for traffic with differing QoS requirements.

- The "Guaranteed Bit Rate" (GBR) is the bit rate that the network guarantees (e.g., through the use of an admission control function) it can sustain for that bearer.

- The "Physical Resource Block" (PRB) is the basic time-frequency resource available for data transmission in LTE. It is equal to $180 \mathrm{kHz}$ per transmission time interval (TTI) [4]

- The "Service Level Agreements" (SLA) between the operators and users is a contract which states the agreed characteristics for each level of service like promised bandwidth and corresponding bill. We propose tree level (highest, medium, lowest) of service GBR ( GBR $\left._{\text {SLA }}\right)$.

\subsection{Proposed AC scheme}

We assume that each customer can request one or more mobile services; we denote session the grouping of all his applications. He can start his session using one media and later on ask for other services which will be assigned if possible to his session. When the traffic is busy and eNB resources are not available, we classify the new request on two groups:

- The first group specifies if the request is a handover session where the connection of the mobile user switches between two eNBs. 
International Journal of Computer Science \& Information Technology (IJCSIT) Vol 3, No 6, Dec 2011

- The second group specifies two types of requests. The first type is a request for a new session when the customer is not connected. The second type is a new media related to an already existing session because we begin by adapting the media to this existing session.

Comparing to classical scheme, when traffic is busy as mentioned in figure 1, we propose adaptations according to request group and type. We preempt resources from services having the most relative utility in GBR $\left(\mathbf{R U}_{\mathbf{G B R}}=\left[\mathbf{G B R} \mathbf{R}_{\text {Served }} / \mathbf{G B R} \mathbf{R}_{\text {SLA }}\right]\right)$. unlike existing work [11] which preempt resources from lowest priority service, we prempt resources from highest $\mathbf{R} \mathbf{U}_{\mathbf{G B R}}$ service.

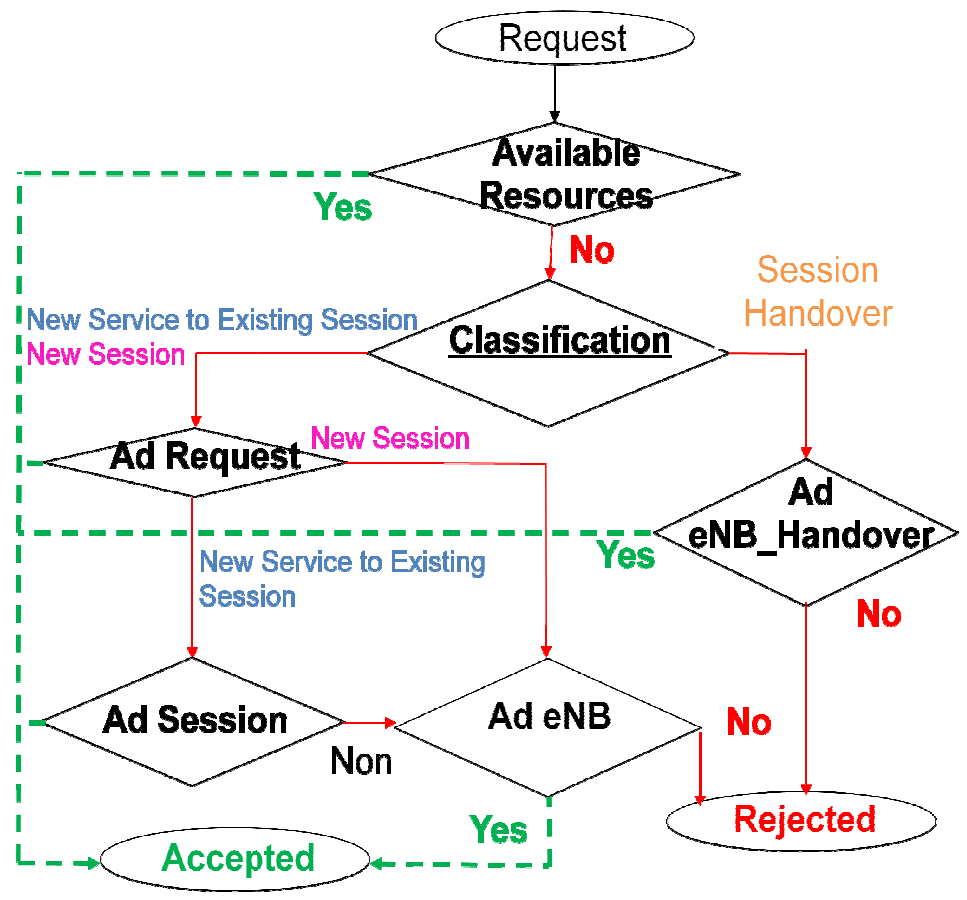

Figure 2. AC Scheme Proposed

Like classical AC, if resources are available our algorithm admits new requests and bandwidth allocated to this request is more or equal to $G R_{\text {SLA }}$. In other case we don't reject media immediately. We propose some types of adaptations:

- If request is not a handover session, first we make SLA adaptation (Ad Request) to request. Our 'Ad Request' algorithm stars reducing the requirement of the new request. This decrease is made only by switching from SLA levels.

- After this step of SLA adaptation, if the problem persists 'Ad Session' algorithm will try to preempt resources from applications which belong to the request session.

- Then, if there is still no resources available, 'Ad eNB' will look for preempting resources from all connected service to eNB.

- If request is e handover session, we don't become by reducing requirement of request because this type is already connected to system. We seek the highest $\mathbf{R U}_{\mathbf{G B R}}$ from both services of request and service of eNB to preempt resources. 
International Journal of Computer Science \& Information Technology (IJCSIT) Vol 3, No 6, Dec 2011

\subsection{Proposed AC Algorithms}

Before presented our algorithms, we define notations used by our algorithms

- $C:$ Total bandwidth capacity of eNB

- $N_{\text {total }}:$ PRB number of eNB

- $K:$ Instantaneous total number of admitted users

- $n_{i}$ : Instantaneous total number of media connected to session number $\mathrm{i}$

- $X_{i}^{j}: \mathrm{X}$ of media number $\mathrm{j}$ and session number $\mathrm{i}$

$\mathrm{X}$ can be:

- $\quad b:$ Bandwidth allocated

- GBR : Guaranteed Bit Rate

- $\quad N$ : PRB number

- $\quad S L A:$ Level Agreement Service

- QCI : QoS Class Identifier

- $\quad B_{a d p}:$ Bandwidth adapted in function by QCI and SLA level

- $\quad R U$ : Relative Usefulness

If new request is a new media of existing session, our algorithm begins by reducing the requirement of the new request (Media Adaptation), section XX explains how make reduce. After this adaptation if problem persist User Adaptation try to preempt resources from applications belong to this session, the preemption techniques will be presented later. When the new request is a new session or handover session, Session adaptation begins by reducing requirement of some or all session's applications. Then if resources are not yet available, eNB Adaptation try to preempt resources from existing sessions.

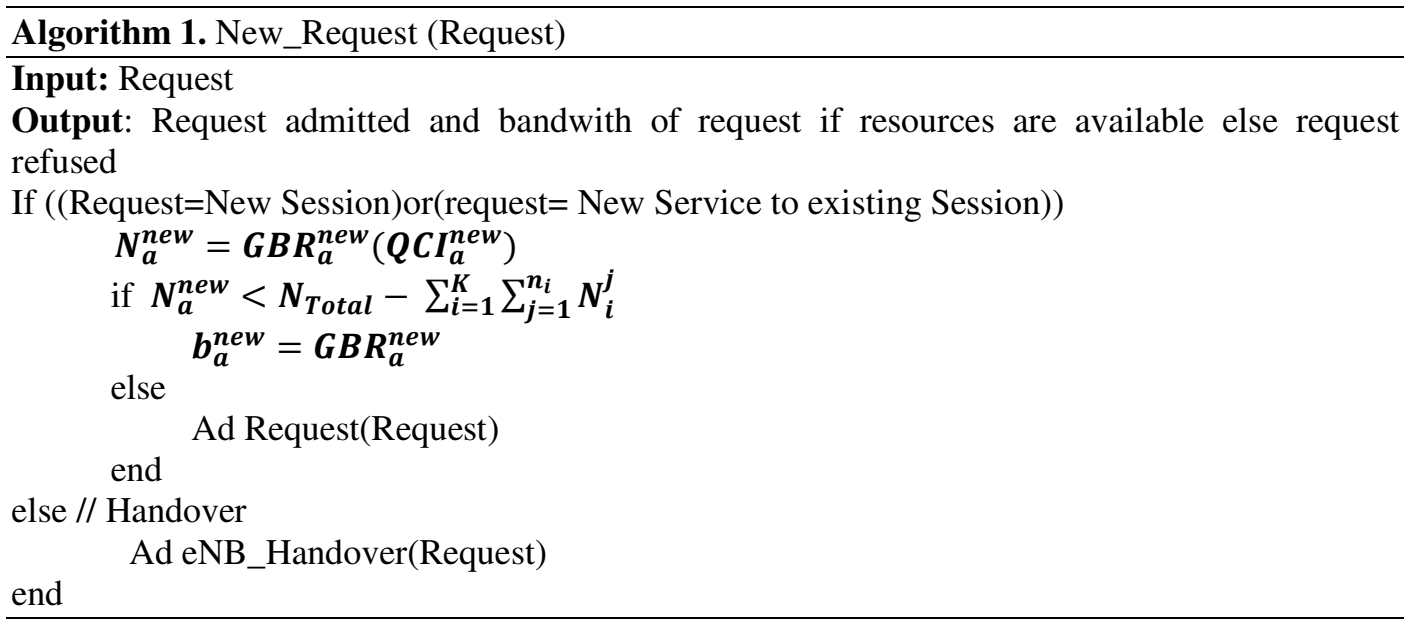

Algorithm 2.Ad Request (Request)

Input: Request 
International Journal of Computer Science \& Information Technology (IJCSIT) Vol 3, No 6, Dec 2011

Output: Request admitted and bandwith of request if resources are available else request refused

Begin

repeat

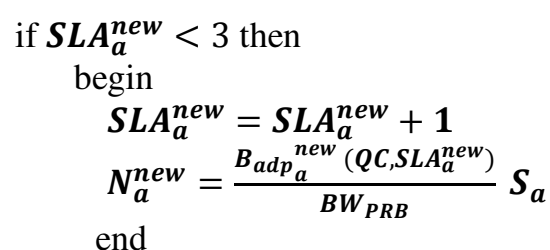

end
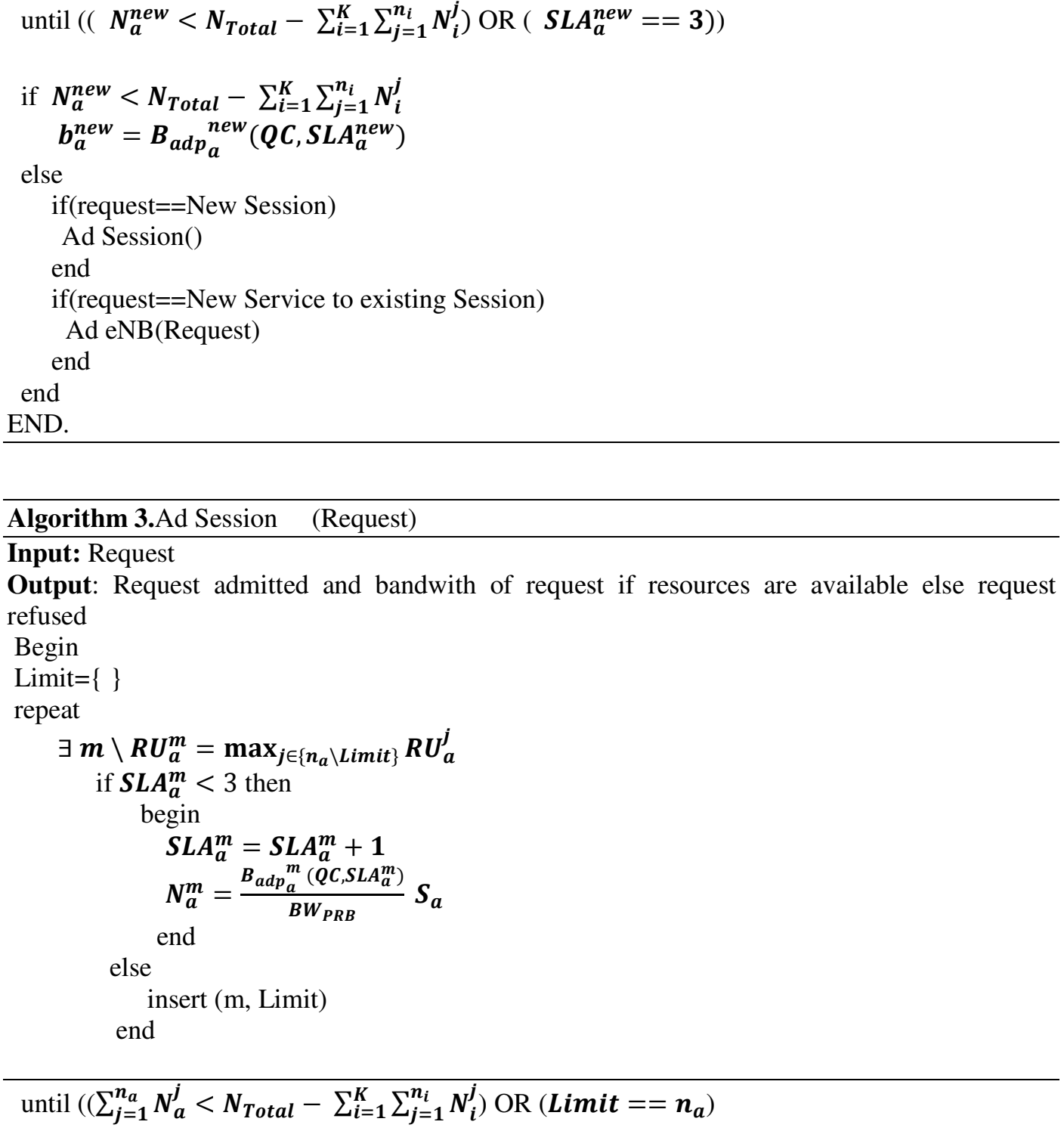
International Journal of Computer Science \& Information Technology (IJCSIT) Vol 3, No 6, Dec 2011

$$
\begin{aligned}
& \text { if } \sum_{j=1}^{n_{a}} \boldsymbol{N}_{a}^{j}<N_{\text {Total }}-\sum_{i=1}^{K} \sum_{j=1}^{n_{i}} N_{i}^{j} \\
& \text { for j=1 to } n_{a} \text { do } \\
& b_{a}^{j}=B_{a d p} p_{a}^{j}\left(Q C, S L A_{a}^{j}\right) \\
& \text { end }
\end{aligned}
$$

else

Ad eNB()

end

END.

Algorithm 4. Ad eNB (Request)

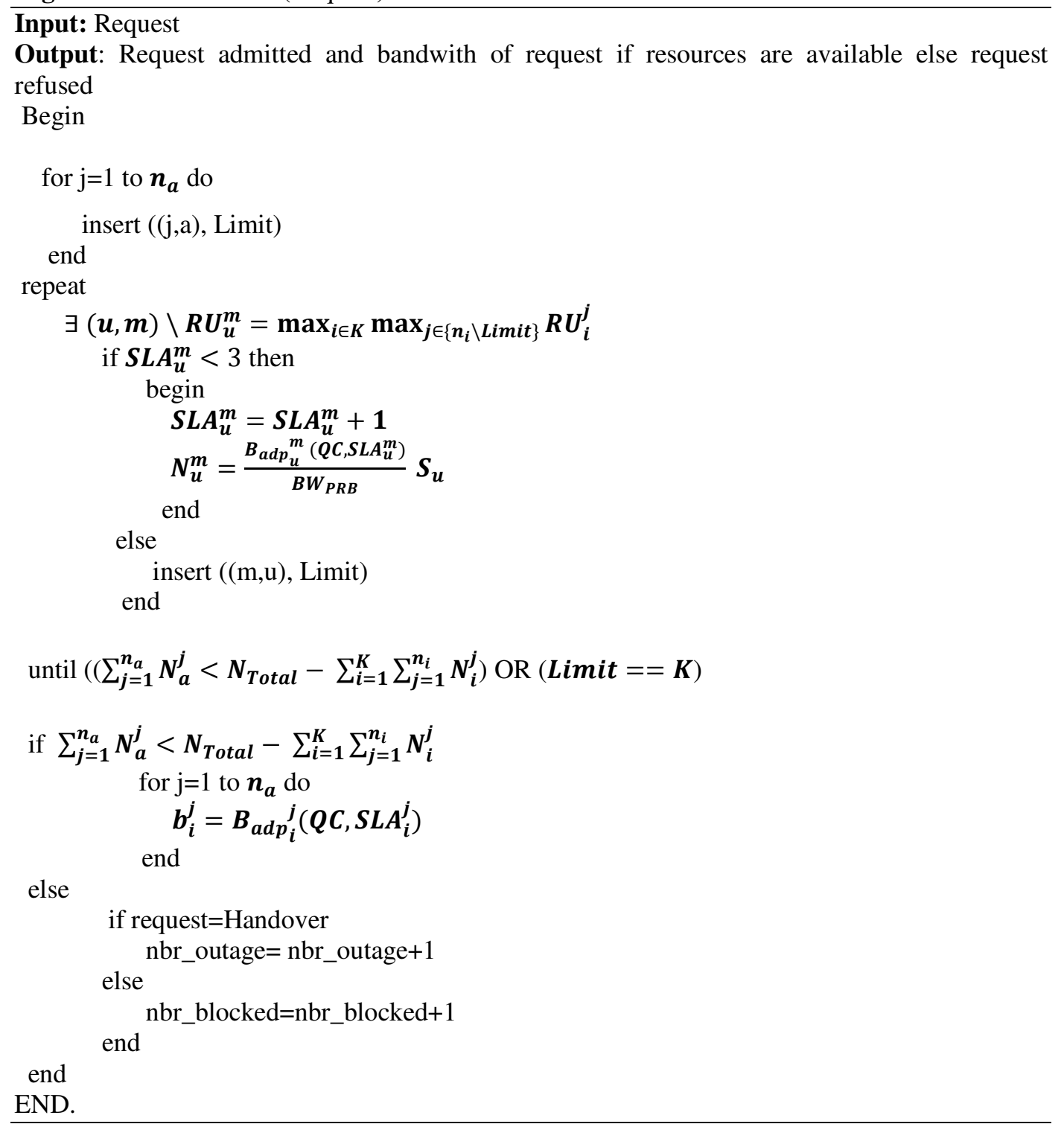


International Journal of Computer Science \& Information Technology (IJCSIT) Vol 3, No 6, Dec 2011

\section{EXPERIMENTAL RESULTS}

We work using an eNB with a total number of PRB equal to 100 and we generate $\mathrm{N}$ request:

- $\mathrm{N}=[50,100,150,200,250,300]$.

- $\quad \mathrm{N}$ is distributed in $\alpha$ new request and $\beta$ handover request.

- We consider four classes of service $C_{\text {num }}$; num=1,2,3,4.

- We have tree level of SLA. SLA level; level=1,2,3.

$$
N=\sum\left[\left(\alpha_{\text {num,level }}+\beta_{\text {num,level }}\right) * C_{\text {num }_{S L A_{\text {level }}}}\right]
$$

Then we applied tree algorithm:

- algo1: the classical admission control algorithm

- $\quad$ algo2 : we add SLA level without adaptations

- $\quad$ algo3: we add SLA level with adaptations

With our solution algo 2 and algo3, the figure 3 shows decrease in blocking rate, figure 4 presents improvement of outage rate and figure 5 indicates improvement in system dependability .

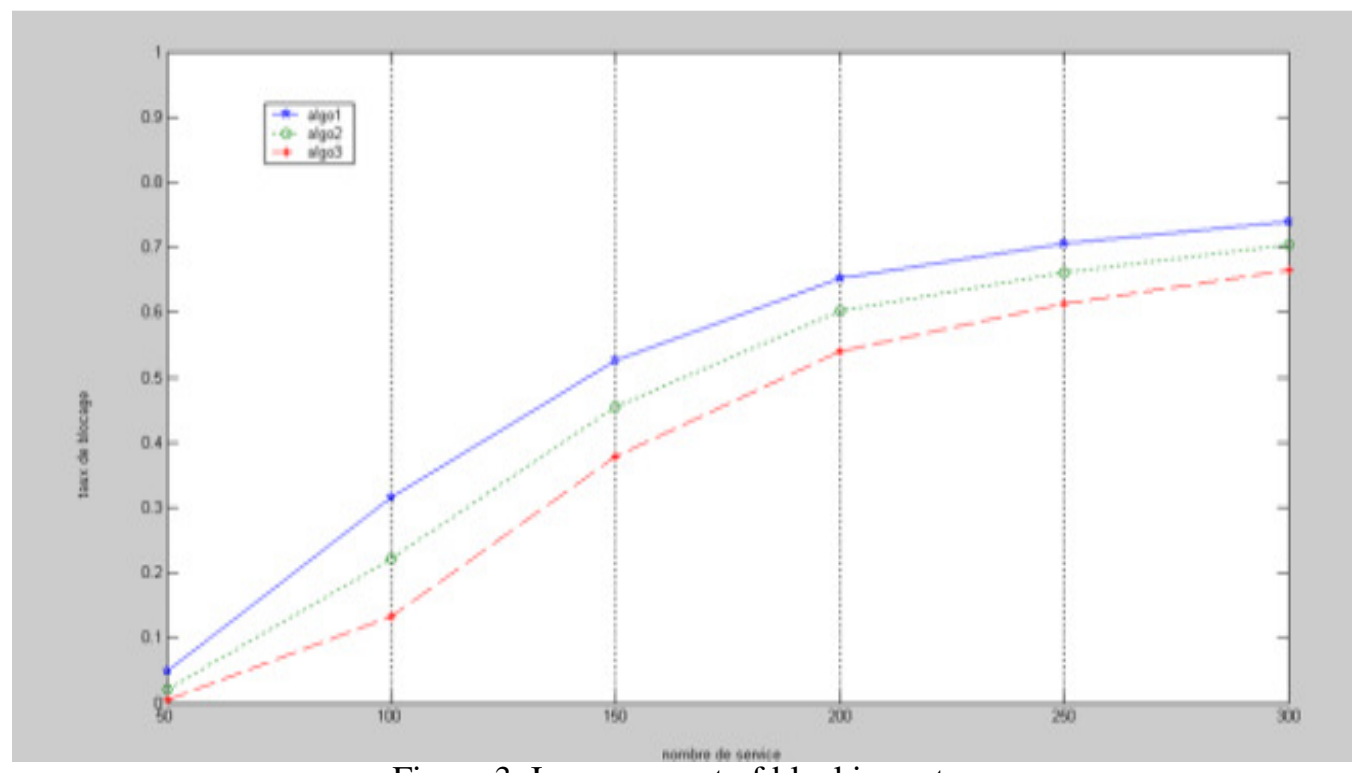

Figure 3. Improvement of blocking rate 


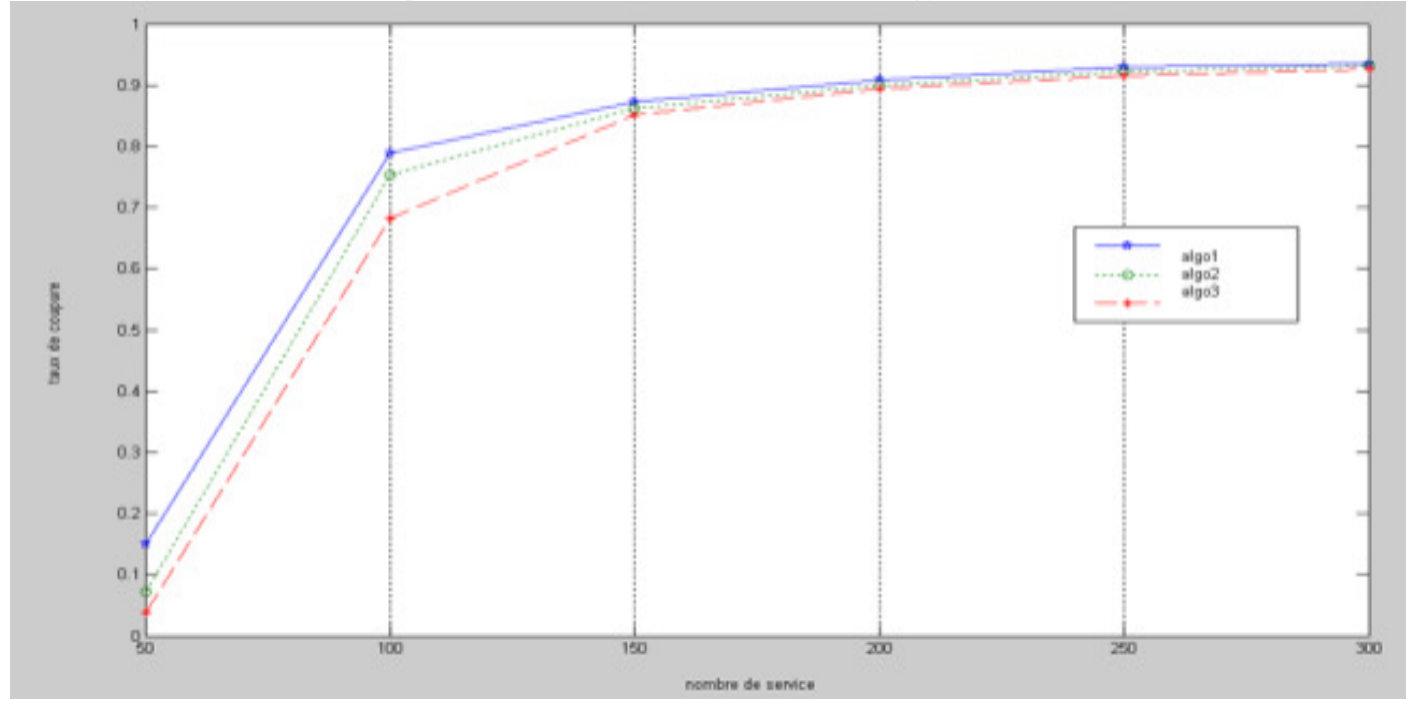

Figure 4. Improvement of outage rate

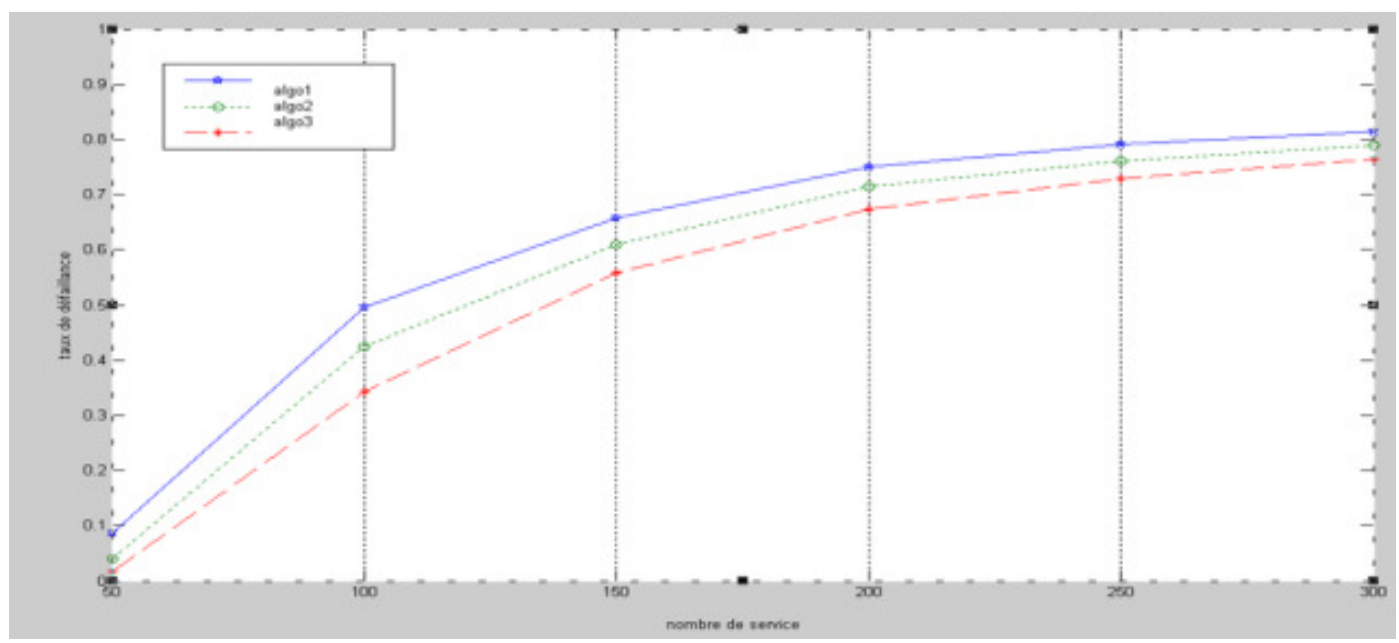

Figure 4: Decrease of failure rate

\section{CONClusion}

According to estimation of increasing mobile application's consumers. We are interested in Long Term Evaluation (LTE) network. LTE dependability depends on availability and reliability of his evolved Nodes Bases stations (eNB). One eNB is available when it is able yet to admit a new media and to support connection. And it is reliable when it is able to support handover connection. In this paper, a utility adaptation for AC algorithm in UTRAN LTE is proposed. In busy network, our approach preempts resources by utility factor to provide new application or to support handover service. Our solution improves the accepted number of user's applications and customer's satisfaction value. Some existing work considers a higher QoS with lower dependability (availability and reliability). We propose solution which improves dependability with providing acceptable QoS. Additional work is needed to improve mobile service dependability from transmitter to receiver. We will try to find solution to improve reliability of services in Core network. 
International Journal of Computer Science \& Information Technology (IJCSIT) Vol 3, No 6, Dec 2011

\section{REFERENCES}

[1] Khalil Laghari, Imen G. Ben Yahia \& Noel Crespi, (2009) "Analysis of Telecommunication Management Technologie”, International Journal IJCSIT, Vol. 1, No. 2.

[2] Emna Abdelfattah \& Asif Mohiuddin, (2010) "Perfermance Analysis of Multimedia Compression Algorithms”, International Journal IJCSIT, Vol. 2, No. 5.

[3] Cisco Visual Networking Index, (2010) "Forecast and methodology 2009-2014", $\underline{\text { http://www.cisco.com/ }}$

[4] Zhaoxin Lu, Hui Tian, Qiaoyun Sun Bo Huang \& Shuqin Zheng, (2010) “An Admission Control Strategy for Soft Frequency Reuse Deployment of LTE Systems”, $7^{\text {th }}$ IEEE CCNC.

[5] S. Sayes, (2009), Comparison betwen WiMax and 3 GPP LTE, Master Thesis.

[6] A. Snow, P. Rastog, \& G. Weckman, (2005) “Assessing Dependability Of Wireless Networks Using Neural Networks", IEEE MILCOM.

[7] J.C Laprie, J.C, (1998) Guide de la sûreté de fonctionnement, Cépadus-éditions.

[8] P.G.S James, H. David, K. Ç. Egemen, J. Abdul, P.R Justin, S. Marcus \& S. Paul, (2010) "Resilience and survivability in communication networks: Strategies, principles, and survey of disciplines", ScienceDirect Journal.

[9] M. Anas, C. Rosa, F.D Calabrese, P.H Michaelsen, K.I Pedersen \& P.E Mogensen, (2008) "QoS-Aware Single Cell Admission Control for UTRAN LTE Uplink", IEEE T VEH COMMUN.

[10] T. Janevski, (2003) Traffic analysis and design of wireless IP networks, ISBN 1-58053-331-0.

[11] M. Qian, Y. Huang, J. Shi, Y. Yuan, L. Tian \& E. Dutkiewicz, (2009) "A Novel Radio Admission Control Scheme for Multiclass Service in LTE Systems”, IEEE GLOBECOM.

[12] O. Ben Ahmed, Z. Choukair \& S. Ben Rejeb, (2010) "User Centric Admission Control Algorithm", GRES conference. 